\title{
Prevention of coprophagy does not alter the hypocholesterolaemic effects of oat bran in the rat
}

\author{
BY KATHRYN A. JACKSON AND DAVID L. TOPPING* \\ CSIRO Division of Human Nutrition, Glenthorne Laboratory, Majors Road, O'Halloran Hill, \\ SA 5159, Australia
}

(Received 10 April 1992 - Accepted 2 October 1992)

\begin{abstract}
Male rats were fed on either a non-purified rodent diet (JS) or cholesterol-free purified diets containing wheat bran (WB) or oat bran (OB). Some animals were allowed normal access to their faeces for coprophagy (coprophagy + ), while in others coprophagy was prevented by placement of a plastic cup over the anus (coprophagy -). Direct ingestion of faeces from the anus was observed in the former groups. Food intake was unaffected by diet or coprophagy status and body weight gain was unchanged with $\mathrm{OB}$ and JS - but was significantly lower with WB - . Plasma cholesterol was highest with WB and equally lower with $\mathrm{OB}$ and JS and was unaffected by coprophagy status. Plasma triacylglycerols were highest with $O B$ and were unaffected by coprophagy status. Caecal digesta mass was highest with JS, intermediate with $O B$ and lowest with WB. Digesta mass was unaffected by coprophagy status with WB and JS but was higher with $\mathrm{OB}$ - . Digesta moisture content was lowest with WB + but highest with WB - . Digesta volatile fatty acid (VFA) concentrations were similarly lower with $\mathrm{OB}+$ and $\mathrm{OB}-$, but were significantly lower with $\mathbf{J S}-$ and WB - than in the corresponding coprophagy + group. In all groups digesta butyrate concentrations were reduced by coprophagy prevention. Pools of total VFA, acetate and butyrate in the digesta were highest with JS. Pools of total VFA in digesta were highest with $\mathrm{JS}+, \mathrm{OB}+$ and $\mathrm{OB}-$ and lowest with $\mathrm{WB}+$ and $\mathrm{WB}-$. The propionate pool was highest with $\mathrm{OB}-$, intermediate with $\mathrm{OB}+$, and equally low in all other groups. The pool of butyrate was highest with JS + and lowest with $\mathrm{OB}-$. Effects of oats and wheat on plasma cholesterol in the rat do not seem to be mediated through faecal re-ingestion.
\end{abstract}

Coprophagy: Plasma lipids: Volatile fatty acids: Rat

Plant foods such as oat bran which are rich in water-soluble non-starch polysaccharides (NSP) lower plasma cholesterol in humans and experimental animals (Judd \& Truswell, 1981 ; Kirby et al. 1982; Illman et al. 1991). The mechanism of this reduction is not entirely certain but probably reflects altered digestion and absorption of fats and steroids (Topping, 1991). Much of the investigative work on the mechanism of cholesterol lowering has been carried out in the rat, a species in which the physiology of the hind gut is rather different to that in humans (Graham \& Aman, 1982). In addition to being a caecal fermenter, the rat is a faecal refector and relies on coprophagy for the supply of B-group vitamins, vitamin $\mathrm{K}$ and essential fatty acids (Barnes, 1962). These nutrients are synthesized by the hind gut microflora but are absorbed most efficiently in the small intestine. To facilitate this absorption, rats ingest stools directly from their anus with about $30-65 \%$ of faeces being recycled directly (Cree et al. 1986).

Effects of coprophagy on plasma cholesterol in the rat have not been investigated, neither has the role of faecal refection on the concentrations and molar proportions of volatile fatty acids (VFA) in the large bowel, although the practice does not seem to modify total fibre digestion by hind gut micro-organisms (Cree et al. 1986). VFA are produced by bacterial

\footnotetext{
* For reprints.
} 
Table 1. Composition ( $\mathrm{g} / \mathrm{kg}$ diet) of diets containing wheat bran (WB) or oat bran $(O B)$

\begin{tabular}{lrrr}
\hline \hline & Diet... & WB & OB \\
\hline Casein & $173 \cdot 3$ & $113 \cdot 3$ \\
Sucrose & $458 \cdot 8$ & $242 \cdot 0$ \\
Maize starch & $136 \cdot 0$ & - \\
Maize oil & $32 \cdot 5$ & - \\
Wheat bran* & $169 \cdot 3$ & - \\
& Oat bran* & - & $661 \cdot 0$ \\
& DL-Methionine & $3 \cdot 0$ & $3 \cdot 0$ \\
& Choline chloride & $1 \cdot 1$ & $1 \cdot 1$ \\
& Vitamin-mineral mixture† & $45 \cdot 0$ & $45 \cdot 0$ \\
\hline
\end{tabular}

* Wet weight.

$\dagger$ AIN-76A vitamin and mineral mixtures purchased from ICN Biomedicals (Costa Mesa, CA, USA)

fermentation of NSP (and other carbohydrates; Fleming \& Arce, 1986) and those NSP that lower cholesterol also appear to be fermented most extensively (Topping, 1991). The present experiments were carried out to ascertain whether prevention of coprophagy modified the response of plasma lipids and caecal VFA to oat and wheat bran and to a standard laboratory rat diet.

\section{MATERIALS AND METHODS}

Animals and diets. Adult male rats of the Hooded Wistar strain were used and were housed under the standard conditions of this laboratory, i.e. in groups of six in stainless-steel cages with wire-mesh bottoms in a room of controlled heating and lighting (Illman et al. 1991). They were allowed free access to water and a cereal-based commercial diet (JS; Joint Stock Diet for Rodents, Milling Industries, Dry Creek, South Australia) before transfer to the experimental diets. All the experimental procedures described were approved by the Animal Care and Animal Ethics Committees of the Division of Human Nutrition according to established guidelines for the care and use of laboratory animals.

Wheat bran and oat bran were obtained from George Weston Foods (Enfield, NSW 2136, Australia). The wheat bran and oat bran were analysed for starch, NSP and lignin by modifications of the methods of Englyst \& Cummings (1988) and Theander \& Westerlund (1986). Fat was determined by extraction and protein was measured as $\mathrm{N}$ using an automated analyser with a conversion factor of 6.25 (Illman et al. 1991). The diet was based on AIN 76A (American Institute of Nutrition, 1977) and was formulated to contain $75 \mathrm{~g} \mathrm{NSP}+$ lignin $/ \mathrm{kg}$ (Table 1). Corrections were made in the specified additions of maize starch, maize oil and casein to allow for starch, fat and protein in the wheat and oat bran. Oat bran was found to contain $(\mathrm{g} / \mathrm{kg})$ : moisture 100, NSP + lignin 126, starch 434, protein 114 , fat 125 , while wheat bran contained $(\mathrm{g} / \mathrm{kg})$ : moisture 110 , NSP + lignin 498, starch 83 , protein 138, fat 103. JS diet is formulated from wheat, wheat pollard, soya-bean meal, meat meal, fish meal and lucerne (Medicago sativa) and contains added vitamins and minerals (Illman \& Topping, 1985).

In some animals (coprophagy-) access to the stool was prevented by the attachment of a pre-weighted plastic cup to the tail with surgical tape so as to cover the anus and retain all faeces. This procedure was developed by modifications of the methods of Wang \& Peters (1963) and Armstrong \& Softly (1966). The cup was weighed and the contents removed at least once daily and the cup was repositioned every morning and evening. In other animals 
Table 2. Food intake ( $g /$ rat per $d)$ and body-weight gain $(g)$ in rats fed on a non-purified diet $(J S)$ or a purified diet containing oat bran $(O B)$ or wheat bran $(W B)$ with $(+)$ or without $(-)$ coprophagy allowed*

(Mean values with pooled standard errors for six observations per group)

\begin{tabular}{ccccc}
\hline Diet & Coprophagy & Food intake & Wt gain \\
\hline JS & + & $27^{\mathrm{a}}$ & $40^{\mathrm{a}}$ \\
& - & $26^{\mathrm{a}}$ & $35^{\mathrm{a}, \mathrm{b}}$ \\
OB & + & $27^{\mathrm{a}}$ & $45^{\mathrm{a}}$ \\
& & - & $27^{\mathrm{a}}$ & $43^{\mathrm{a}}$ \\
WB & + & $26^{\mathrm{a}}$ & $42^{\mathrm{a}}$ \\
& & $25^{\mathrm{a}}$ & $26^{\mathrm{b}}$ \\
& & 2 & 5 \\
\hline
\end{tabular}

$\mathrm{a}, \mathrm{b}$ In any column, mean values with unlike superscript letters were significantly different $(P<0.05)$.

* For details of diets and procedures, see Table 1 and pp. 212-213.

(coprophagy +) plastic cups were taped to the tail so as to allow normal faecal refection. Faeces were collected from beneath the cages of these animals for $48 \mathrm{~h}$ on two separate occasions and weighed. Cups were checked daily at 09.00 hours and then at intervals of 2-3 h. There were six groups (each of six animals): oat bran with $(\mathrm{OB}+)$ and without $(\mathrm{OB}-)$ coprophagy, wheat bran with $(\mathrm{WB}+)$ and without $(\mathrm{WB}-)$ coprophagy, and joint stock with $(\mathrm{JS}+)$ and without (JS - ) coprophagy. During the experimental period the animals were housed in individual cages.

Analytical procedures. After $10 \mathrm{~d}$ of dietary modification the rats were lightly anaesthetized with diethyl ether and blood $(5 \mathrm{ml})$ was drawn from the abdominal aorta and collected into ice-cold tubes containing EDTA (dipotassium salt) as anticoagulant. Plasma was prepared by centrifugation and assayed for total and high-density-lipoprotein (HDL) cholesterol and triacylglycerols (TAG) as described previously (Illman \& Topping, 1985). Whole caeca were excised, the contents extruded and weighed for measurement of moisture content and VFA concentrations (Illman \& Topping, 1985).

Statistical methods. Statistical evaluation was by analysis of variance incorporating a calculation of least significant difference. Data are shown in tabular form as the means with the pooled standard error. A value of $P<0.05$ was taken as the criterion of significance.

\section{RESULTS}

Food consumption, body-weight gain and faecal wet weight. The animals appeared to find all diets palatable, and food intake was constant over the experimental period with an average intake of $26 \mathrm{~g} /$ rat per d, all groups combined (Table 2). Weight gain was unaffected by diet in any of the groups allowed coprophagy (Table 2). Weight gain was also the same in the $\mathrm{OB}$ - and JS - groups but was significantly lower in the WB - group with a gain that was lower than all other groups except JS - .

Rats in both groups appeared to find the collection cups irritating, particularly at the beginning of the experiment, and made efforts to remove them. Presumably because the cups were further down the tail (and so more accessible), this seemed to be greater for the coprophagy + rats. Cups were removed completely (and the faecal collection was incomplete) on eight occasions by different animals. These removals occurred in all three groups and were mostly in the first $6 \mathrm{~d}$ of the experiment. Full faecal collections were obtained on all other occasions. The average output for the whole experiment was $11 \cdot 7,5 \cdot 6$ 
Table 3. Concentrations of plasma total and high-density-lipoprotein $(H D L)$ cholesterol and triacylglycerol (TAG) in rats fed on a non-purified diet $(J S)$ or a purified diet containing oat bran $(O B)$ or wheat bran $(W B)$ with $(+)$ or without $(-)$ coprophagy allowed*

(Mean values with pooled standard errors for six observations per group)

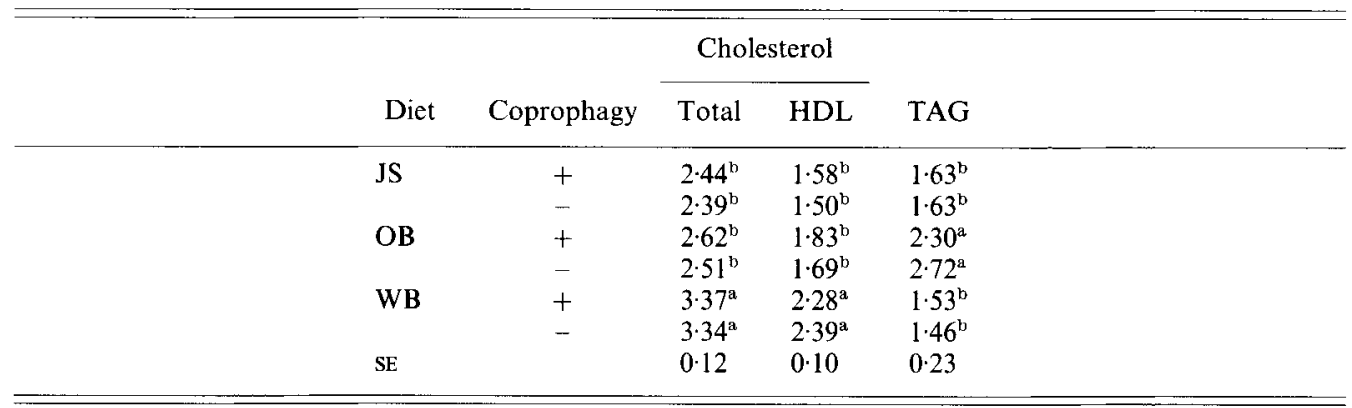

${ }^{\mathrm{a}, \mathrm{b}}$ In any column, values with unlike superscript letters were significantly different $(P<0.05)$.

* For details of diets and procedures, see Table 1 and pp. 212-213.

Table 4. Caecal digesta wet weight $(\mathrm{g})$ and moisture content $(\mathrm{g} / \mathrm{kg})$ of rats fed on a nonpurified diet $(J S)$ or a purified diet containing oat bran $(O B)$ or wheat bran $(W B)$ with $(+)$ or without $(-)$ coprophagy allowed ${ }^{*}$

(Mean values with pooled standard errors for six observations per group)

\begin{tabular}{ccccc}
\hline \hline & Diet & Coprophagy & Wet wt & Moisture \\
\hline IS & + & $4 \cdot 98^{\mathrm{a}}$ & $710^{\mathrm{b}, \mathrm{c}}$ \\
& - & $4 \cdot 70^{\mathrm{a}}$ & $710^{\mathrm{b}, \mathrm{c}}$ \\
OB & + & $3 \cdot 16^{\mathrm{c}}$ & $780^{\mathrm{a}, \mathrm{b}}$ \\
& - & $3 \cdot 97^{\mathrm{b}}$ & $750^{\mathrm{a}, \mathrm{b}}$ \\
& WB & + & $2 \cdot 69^{\mathrm{c}}$ & $660^{\mathrm{c}}$ \\
& - & $2 \cdot 62^{\mathrm{c}}$ & $820^{\mathrm{a}}$ \\
& & $0 \cdot 27$ & 40 \\
\hline
\end{tabular}

$a, b, c$ In any column, values with unlike superscript letters were significantly different $(P<0.05)$,

* For details of diets and procedures, see Table 1 and pp. 212-213.

and 4.3 (pooled SE $0 \cdot 2, n$ 122) for JS, OB and WB respectively. In comparison, the collections were $6 \cdot 6,4 \cdot 4$ and $3 \cdot 2$ (pooled SE $0 \cdot 3, n 36$ ) for the corresponding animals in which coprophagy was allowed.

Plasma cholesterol and TAG. Plasma total cholesterol was highest in rats fed on diet WB and equally lower with OB and JS (Table 3). Similar differences were seen with HDL cholesterol, with higher concentrations in the WB group than in the other two groups. Neither total nor HDL cholesterol was affected by coprophagy status. Plasma TAG was significantly higher with OB than with JS and WB, and in no group was there any effect of coprophagy prevention (Table 3 ).

Caecal digesta and VFA. Caecal digesta wet weight was the highest in the JS + and groups and was significantly lower in the OB - group (Table 4). This latter weight was significantly higher than in $\mathrm{OB}$ - rats. Digesta wet weight did not differ between $\mathrm{OB}-$, $\mathrm{WB}+$ and $\mathrm{WB}-$ animals. Digesta moisture content was highest in the WB - and $\mathrm{OB}-$ groups but only the former was significantly higher than JS +, JS - , and $\mathrm{OB}-$. Moisture content in the WB + group was the same as in JS + and JS - animals. 
Table 5. Concentrations (mmol/l) of total and individual volatile fatty acids $(V F A)$ in caecal digesta of rats fed on a non-purified diet $(J S)$ or a purified diet containing oat bran $(O B)$ or wheat bran $(W B)$ with $(+)$ or without $(-)$ coprophagy allowed*

(Mean values with pooled standard errors for six observations per group)

\begin{tabular}{ccccccc}
\hline Diet & Coprophagy & Acetate & Propionate & Butyrate & Total VFA \\
\hline JS & + & $102^{\mathrm{a}}$ & $27^{\mathrm{b}}$ & $51^{\mathrm{a}}$ & $188^{\mathrm{a}, \mathrm{b}}$ \\
& - & $49^{\mathrm{b}}$ & $17^{\mathrm{b}}$ & $29^{\mathrm{a}, \mathrm{b}}$ & $101^{\mathrm{c}}$ \\
OB & + & $95^{\mathrm{a}}$ & $88^{\mathrm{a}}$ & $37^{\mathrm{a}}$ & $224^{\mathrm{a}}$ \\
& - & $92^{\mathrm{a}}$ & $99^{\mathrm{a}}$ & $6^{\mathrm{b}}$ & $197^{\mathrm{a}, \mathrm{b}}$ \\
WB & + & $87^{\mathrm{a}, \mathrm{b}}$ & $24^{\mathrm{b}}$ & $48^{\mathrm{a}}$ & $164^{\mathrm{a}, \mathrm{b}, \mathrm{c}}$ \\
& - & $69^{\mathrm{a}, \mathrm{b}}$ & $23^{\mathrm{b}}$ & $33^{\mathrm{a}}$ & $128^{\mathrm{b}, \mathrm{c}}$ \\
& & 20 & 13 & 13 & 41 \\
\hline
\end{tabular}

u, b. In any column, values with unlike superscript letters were significantly different $(P<0-05)$.

* For details of diets and procedures, see Table 1 and pp. 212-213.

Table 6. Pools (umol) of total and individual volatile fatty acids (VFA) in caecal digesta of rats fed on a non-purified diet (JS) or a purified diet containing oat bran $(O B)$ or wheat bran $($ WB) with $(+)$ or without $(-)$ coprophagy allowed*

(Mean values with pooled standard errors for six observations per group)

\begin{tabular}{cccccc}
\hline Diet & Coprophagy & Acetate & Propionate & Butyrate & Total VFA \\
\hline JS & + & $365^{\mathrm{a}}$ & $97^{\mathrm{b}, \mathrm{c}}$ & $189^{\mathrm{a}}$ & $680^{\mathrm{a}}$ \\
& - & $166^{\mathrm{c}}$ & $58^{\mathrm{c}}$ & $99^{\mathrm{b}}$ & $339^{\mathrm{b}, \mathrm{c}}$ \\
OB & + & $232^{\mathrm{b}, \mathrm{c}}$ & $218^{\mathrm{b}}$ & $88^{\mathrm{b}}$ & $544^{\mathrm{a}, \mathrm{b}}$ \\
& - & $276^{\mathrm{b}}$ & $296^{\mathrm{a}}$ & $19^{\mathrm{c}}$ & $590^{\mathrm{a}}$ \\
WB & + & $152^{\mathrm{c}}$ & $43^{\mathrm{c}}$ & $86^{\mathrm{b}, \mathrm{c}}$ & $288^{\mathrm{c}}$ \\
& - & $147^{\mathrm{c}}$ & $47^{\mathrm{c}}$ & $69^{\mathrm{b}, \mathrm{c}}$ & $286^{\mathrm{c}}$ \\
& & 52 & 37 & 34 & 107 \\
\hline
\end{tabular}

a. B.e In any column, values with unlike superscript letters were significantly different $(P<0 \cdot 05)$.

* For details of diets and procedures, see Table 1 and pp. 212-213.

The concentrations of total VFA were similar in JS,$+ \mathrm{OB}+$ and $\mathrm{WB}+$ rats and were unaffected by prevention of faecal refection with diets $O B$ and WB (Table 5). VFA concentrations were significantly lower in JS,$- \mathrm{OB}+$ and $\mathrm{WB}+$ rats than in the corresponding animals in which coprophagy was allowed. Acetate was the major VFA in animals fed on WB and JS but with $\mathrm{OB}+$ propionate was present at the same concentration. In the two former groups, butyrate was the next major acid. Prevention of faecal refection did not change acetate or propionate concentrations with the OB diet but did give very much lower butyrate. With WB there were no major changes in individual acids but with JS, there were major falls in acetate, propionate and butyrate.

These changes in VFA concentration with diet and treatment do not reflect fully the situation in the colon due to variations in digesta mass. Calculation of the pool size of VFA (i.e. concentration $\times$ fluid volume) showed that the largest pool of total VFA was with JS + and $\mathrm{OB}+$ and lowest with WB + and WB - although these latter groups did not differ to JS - (Table 6). The pool in OB + was similar to that in JS +, JS - and OB + rats. As acetate was the major acid, differences in its pool size tended to mirror those in total VFA. 
In the case of propionate, OB - had the largest pool and JS - , WB - and WB had the smallest. Butyrate was highest in the JS + group and lowest with $\mathrm{OB}-$ although this was not significantly different to $\mathrm{WB}+$ and $\mathrm{WB}-$.

\section{DISCUSSION}

The rats used in these studies were kept in cages with wire-mesh bottoms which are the standard conditions of this laboratory. Under these circumstances direct ingestion of faeces from the anus is the only feasible means for significant faecal re-ingestion and has been observed previously and currently in rats in which coprophagy was allowed. Social coprophagy has been observed ( $G$. A. Crook, personal communication) in the common marmoset (Callithrix jacchus), another species housed at these laboratories. This is a possibility in rats kept in groups but could not have occurred in the present study as the rats were housed individually. Our aim in the present experiment was to test the effect of prevention of coprophagy rather than coprophagy itself so stools were collected only on two occasions in coprophagy + rats. The average stool output in coprophagy + rats was the same in both collections and was greatest with JS and least with OB. In the coprophagy - animals, output was highest in the JS - groups and about $70 \%$ higher than JS + animals. With WB the differences were relatively slight, but again with $\mathrm{OB}$ there was a $60 \%$ difference. Inter alia these findings indicate that prevention of coprophagy did lead to an apparent increase in stool output consistent with prevention of faecal refection, and that the increase depended on diet. However, a note of caution must be added in that these were limited collections and we did not measure stool water so that we have no information on relative outputs of dry matter (which would be a truer indication of faecal excretion).

As expected from previous studies (e.g. Shinnick et al. 1990; Illman et al. 1991), plasma total and HDL cholesterol were significantly higher with WB than with OB. It might be argued that these differences reflect compensatory differences in the additions of other dietary components (e.g. sucrose) to balance those present in the brans. However, the accumulated evidence indicates that the differences are due to bran components. Thus, higher plasma cholesterol concentrations appear to be a consistent finding with wheat bran in the rat (Asp et al. 1981), which accords with studies in humans. Similarly, feeding oat bran results in lower cholesterol in both rats and humans. In rats the greater effect seems to be on HDL whereas in humans the greatest reduction with oat bran is in low-densitylipoprotein (LDL) cholesterol (Kestin et al. 1990), which seems to reflect the relative importance of the HDL as a transporter of plasma cholesterol in the rat (Arbeeny et al. 1987). It does seem that in both rats and humans the mechanisms of chelesterol reduction are the same, i.e. altered absorption of fats and/or bile acids and neutral sterol excretion, and it has been documented in animals (Illman \& Topping, 1985) and humans (Zhang et al. 1991) that cereals such as oats and barley which lower cholesterol also enhance faecal sterol excretion. This loss is believed to be responsible for the higher TAG concentrations in rats fed on OB, as bile salt depletion stimulates hepatic TAG secretion and raises plasma concentrations (Nestel \& Grundy, 1976). In the present experiments JS also gave lower plasma cholesterol concentrations than WB which may equate with the oat content of the JS diet. However, this cannot be the full explanation as plasma TAG levels were the same with JS as those with WB. It is known that, in both oats (Illman et al. 1991) and barley (Zhang et al. 1991), components other than soluble NSP are involved in the cholesterol reduction and the differences may reflect the mix of those components in the JS diet. Prevention of coprophagy did not modify the response of the animals to the three experimental diets indicating that their effects did not depend on re-ingestion of fats and steroids in faeces. We had considered this to be an important possibility as both cholesterol, coprostanol and 
primary and secondary bile acids are recycled in this process. Thus, the effects of oats and wheat on plasma lipids in the rat are not a consequence of faecal refection.

Another important area where prevention of coprophagy had no effect was in food intake. As mentioned previously, the rats initially found the collection cups to be an irritant but then became adapted to their presence which may explain the lack of effect on food consumption. Although prevention of faecal refection has been reported to inhibit bodyweight gain (Barnes et al. 1963; Cree et al. 1986), this was not the case with diets JS and $\mathrm{OB}$ in the present studies. However, we did find a significant loss of weight gain with WB which is in keeping with those earlier findings. Coprophagy has the advantage of recovering essential nutrients generated in the caecum but it is equally true that faecal ingestion may be at the expense of food intake (Lutton \& Chevallier, 1973). Thus, it is possible that with an unrestricted food supply diets JS and OB were able to supply all the nutrient needs of the animal. Diet WB, which gave lower VFA concentrations (indicating less fermentation), may have been unable to meet those requirements, although Cree et al. (1986) have reported no effect of coprophagy prevention on apparent fibre digestibility in the rat.

Both diet and experimental treatment had significant effects on digesta water content. Effects of diet have been noted previously but not of coprophagy status. The cause of the higher moisture level in the WB - group is not certain but may be contributory to the weight loss. It is known that VFA stimulate large bowel fluid and electrolyte absorption (Fleming \& Arce, 1986) and it is possible that the lower VFA concentrations in this group were responsible. In animals where coprophagy was allowed, total VFA concentrations were highest with $\mathrm{OB}$ and lowest with WB. Concentrations of acetate were very similar between the three different groups and most of the difference between $O B$ and WB was in propionate with high concentrations in the $O B$ group. This difference has been noted previously (Illman et al. 1991) and it has been proposed that the preferential production of propionate mediates the cholesterol reduction by oat bran as this acid inhibits hepatic cholesterol synthesis (Chen et al. 1984). However, the concentrations of propionate required seem to be quite outside the physiological range (Illman et al. 1988) and in the present experiments both $\mathrm{OB}$ and JS gave similar plasma cholesterol concentrations with propionate concentrations with OB that were $325 \%$ higher than those with JS. Prevention of coprophagy did not affect total VFA or acetate and propionate in the OB group. However, butyrate concentrations were very low in the $\mathrm{OB}$ - animals. Butyrate is regarded as a major metabolic fuel for the cells of the large bowel wall (Roediger, 1982) and its concentrations in digesta are extremely susceptible to nutritional manoeuvres such as starvation or starvation followed by refeeding (Butler et al. 1990). In starved animals butyrate concentrations fall very rapidly due to inadequate production coupled with continued utilization, and presumably in the $\mathrm{OB}$ - animals a similar change must have occurred. In the other groups, butyrate concentrations did not decline as much, suggesting that in those animals production was adequate to meet the demands. However, in the JS - animals there was a very large fall in the concentration of acetate which was not found in the WB or OB animals. This suggests some degree of specificity in the effects of dietary NSP on the gut microflora which is modified by the abolition of coprophagy. It is possible that these specificities may have influenced body-weight gain. Fermentation of NSP and other polysaccharides yields energy as VFA for the host. The exact energy yield for this process has yet to be quantified fully but is less efficient than that for digestion occurring in the small intestine (Livesey, 1990). In both the OB and JS groups weight gain was unaffected by coprophagy status but in the WB - group weight gain was less. As mentioned previously, caecal VFA concentrations were least resistant to change in the WB - rats which may indicate that the same amount of fermentable substrate was reaching the hind gut under both conditions. In animals fed on the other diets the fall in VFA concentration could 
reflect an increase in small intestinal digestive efficiency leading to the passage of less fermentable material to the caecum. However, some caution must be exercised in interpreting the findings entirely in terms of concentration, as the VFA availability must also be assessed as pool size. In this instance the highest pools of total VFA and acetate were found with JS + and the lowest with WB + and WB - . Butyrate also was highest with $\mathrm{JS}+$ and lowest with $\mathrm{OB}-$. We did not measure faecal VFA output (and so cannot comment on any loss) but the caecal pool findings do imply that the smaller pool with WB -- might have contributed to the lower weight gain. Significant differences were found in propionate pool with WB - being again the lowest. Whether this contributed to the higher digesta moisture content in that group remains to be established. However, VFA are known to stimulate large bowel fluid transport and this might be a contributory factor.

\section{K.A.J. thanks George Weston Foods for generous financial support.}

\section{REFERENCES}

American Institute of Nutrition (1977). Report of the American Institute of Nutrition ad hoc committee on standards for nutritional studies. Journal of Nutrition 107, 1340-1348.

Arbeeny, C. M., Rifici, V. A. \& Eder, H. A. (1987). The uptake of the apoprotein and cholesteryl ester of highdensity lipoproteins by the perfused rat liver. Biochimica et Biophysica Acta 917, 9-17.

Armstrong, B, K. \& Softly, A. (1966). Prevention of coprophagy in the rat. A new method. British Journal of Nutrition 20, 595-598.

Asp, N. G., Bauer, H. G., Nilsson-Ehle, P., Nyman, M. \& Oste, R. (1981). Wheat bran increases high-densitylipoprotein cholesterol in the rat. British Journal of Nutrition 46, 385-393.

Barnes, R. H. (1962). Nutritional implications of coprophagy. Nutrition Reviews 20, 289-291.

Butler, R. N., Topping, D. L., Illman, R. J., Goland, G. J., Lawson, M. J. \& Roberts-Thomson, I. C. (1990). Effects of starvation-refeeding on volatile fatty acid distribution in the large bowel of the rat. Nutrition Research 10, 91-98.

Chen, W.-J. L., Anderson, J. W. \& Jennings, D. (1984). Propionate may mediate the hypocholesterolaemic effects of certain soluble plant fibers in cholesterol-fed rats. Proceedings of the Society for Experimental Biology and Medicine 175, 215-218.

Cree, T. C., Wadley, D. M. \& Marlett, J. A. (1986). Effect of coprophagy prevention in the rat on neutral detergent fiber digestibility and apparent calcium absorption. Journal of Nutrition 116, 1204-1208.

Englyst, H. \& Cummings, J. H. (1988). Improved method of measurement of dietary fibre as non-starch polysaccharides in plant food. Journal of the Association of Official Analytical Chemists 71, 808-814.

Fleming, S. E. \& Arce, D. S. (1986). Volatile fatty acids: their production, absorption, utilisation and roles in human health. Clinical Gastroenterology 15, 787-814.

Graham, H. \& Aman, P. (1982). The pig as a model in dietary fibre digestion studies. Scandinavian Journal of Gastroenterology 22, Suppl. 129, 55-61.

Illman, R. J. \& Topping, D. L. (1985). Effects of dietary oat bran on faecal sterol excretion, plasma volatile fatty acids and lipid synthesis in the rat. Nutrition Research 5, 839-846.

Illman, R. J., Topping, D. L., Dowling, K., Trimble, R. P., Russell, G. R. \& Storer, G. B. (1991). Effects of solvent extraction on the hypocholesterolaemic action of oat bran in the rat. British Journal of Nutrition $\mathbf{6 5}$, $435-443$.

Illman, R.J., Topping, D. L., McIntosh, G. H., Trimble, R. P., Storer, G. B., Taylor, M. N. \& Cheng, B.-Q. (1988). Hypocholesterolaemic effects of dietary propionate: studies in whole animals and perfused rat liver. Annals of Nutrition and Metabolism 32, 97-107.

Illman, R. J., Topping, D. L. \& Trimble, R. P. (1986). Effects of food restriction and starvation-refeeding on volatile fatty acid concentrations in the rat. Journal of Nutrition 116, 1694-1700.

Judd, P. A. \& Truswell, A. S. (1981). The effects of rolled oats on blood lipids and faecal steroid excretion in man. American Journal of Clinical Nutrition 34, 2061-2067.

Kestin, M., Moss, R., Clifton, P. M. \& Nestel, P. J. (1990). The comparative effects of three cereal brans on plasma lipids, blood pressure and glucose metabolism in mildly hypercholesterolemic men. American Journal of Clinical Nutrition 52, 661-666.

Kirby, R. W., Anderson, J. W., Sieling, B., Rees, E. D., Chen, W.-J., Miller, R. E. \& Kay, R. M. (1981). Oat-bran intake selectively lowers serum low-density lipoprotein cholesterol concentrations of hypercholesterolemic men. American Journal of Clinical Nutrition 34, 824-829.

Livesey, G. (1990). Energy values of unavailable carbohydrate and diets: an inquiry and analysis. American Journal of Clinical Nutrition 51, 617-637. 
Lutton, C. \& Chevallier, F. (1973). Coprophagy in the white rat : quantitative aspects and time relations with food intake. Journal of Nutrition 66, 219-228.

Nestel, P. J. \& Grundy, S. M. (1976). Changes in plasma triglyceride secretion during withdrawal of bile. Metabolism 25, 1259-1268.

Roediger, W. E. W. (1982). The effect of bacterial metabolites on nutrition and function of the colonic mucosa. Symbiosis between man and bacteria. In Colon and Nutrition, pp. 11-24 [H. Kaspar and H. Goebell, editors] Lancaster, Boston and the Hague: MTP Press Ltd.

Shinnick, F. L., Ink, S. L. \& Marlett, J. A. (1990). Dose response to a dietary oat bran fraction in cholesterol-fed rats. Journal of Nutrition 120, 561-568.

Theander, O. \& Westerlund, E. A. (1986). Studies on dietary fibre. 3. Improved procedures for analysis of dietary fibre. Journal of Agricultural and Food Chemistry 34, 330-336.

Topping, D. L. (1991). Soluble fibre polysaccharides: effects on plasma cholesterol and colonic fermentation. Nutrition Reviews 49, 195-203.

Wang, C. \& Peters, D. (1963). Modification of the anal cup technique for small experimental animals. Laboratory Animal Care 13, 105-108.

Zhang, J.-X., Lundin, E., Andersson, H., Bosaeus, I., Dahlgren, S., Hallmans, G., Stenling, R. \& Aman, P. (1991). Brewer's spent grain, serum lipids and faecal steroid excretion in human subjects with ileostomies. Journal of Nutrition 121, 778-784. 